\title{
AI INFLUENCE IN COVID-19 DETECTION
}

\author{
Cijil Benny
}

MSc Data Science, Department of Mathematics, Chandigarh University, Gharuan-Mohali, Punjab-140413, India.

\begin{abstract}
This paper is on analysing the feasibility of AI studies and the involvement of AI in COVID interrelated treatments. In all, several procedures reviewed and studied. It was on point. The best analysing methods on the studies were Susceptible Infected Recovered and Susceptible Exposed Infected Removed respectively. Whereas the implementation of AI mostly done in X-ray's and CTScans with the help of Convolutional Neural Network. To accomplish the paper several data sets are used. They include medical and case reports, medical strategies and persons respectively. Approaches are being done through shared statistical analysis based on these reports. Considerably the acceptance COVID is being shared and it is also reachable. Furthermore, much regulation is needed for handling this pandemic since it is a threat to global society. And many more discoveries shall be made in the medical field uses AI as primary key source.
\end{abstract}

Keywords: Computed Tomography (CT), Logistic Regression (LR), Linear Discriminant Analysis (LDA), Convolutional Neural Network (CNN), Artificial Intelligence (AI), Random Forest (RF),Naive Bayes (NB), Support Vector Machines (SVM) andDecision Trees (DT).

\section{Introduction}

In a public well-being emergency of international reputation on 30 January 2020, the World Health Organization proclaimed that 2019 new coronavirus (COVID-19) is an international apprehension [1]. Again, 7818 familiarized COVID-19 cases were totally serious, with more than 1370 caustic cases and 170 deaths in whole. The build-up was in upshot in China [2]. Over the subsequent couple of weeks, advertising outside the limits of China contaminates every country. Dry cough, abscessed stomach, and fever are symptoms of illness. While the widely held of cases are moderate, remaining can lead to ARDS, acerbic pneumonia, oedema in the lung and downfall of a body. Once acknowledged by WHO as a matter of emergency many works have been passed out to access adobe and aware in order to try to use floorboards to access either the broadcasting of conditions, to admit defending measures by rules and to analyse an authentic disorder latterly only to name insufficient.

Artificial intelligence (AI) is a forecast account. AI is the adjustment and construction of computers, the abecedary insight of humans. AI has been well-in step in a number of areas, together with machine vision, web publicity, junk screening, computerization, artifice warning etc. In health care, AI has also been able to admittancepain identification, valuation collection, all-round observation, biological study, the footnote of gene deed, automated trials, the gathering of reflex results, etc. [3]. With respect to the Covid applications, AI is enhanced in the procurement, evaluation and calculation of medical archangel. In this article, the report, annual and cautioning of COVID-19 delivers an assessment of the faulty intellectfamiliarized.

\section{AI and COVID}

For medical division and review, the main adaptation was Artificial Intelligence to regulate whether the spaces were COVID-19 or whether the contamination was severely affected. In these works, mostly given in the view of $\mathrm{x}$-rays or computational radiology (CT). A brief summary of these medical imaging methods is given previous presentation of the AI methodology adjusted in the apprehension and sorting of COVID-19. 


\section{COVID detection based on CT scan}

Radiology of X-rays involves of bright x-rays integrating the anatomical credit of the photons to be analysed and the canyon photons to the provision of the body. It can inferior (block) some of the exploit photons liable on the body type of tissue. This keep postedas an anatomical illustration of a detector in the inside of the body. Radiology of the X-ray has been established for bone pathology and for the calculation of lung infections. CT takes X-ray $\mathrm{x}$-ray credentials from various angles of crosssectional post-sectional images after the body is separated into X-ray demographic pictures of the Anatomy. These sectioned pictures, often mentioned to as slices, are tomographic snaps that provide more comprehensive medical guidance than the appropriate x-ray x-rays. In accumulation to defining pulmonary embolization's, equilibrium fluid, and pneumonia in the lungs, CT images can be get used to detect anatomy anomalies such as tumours and drain [4]. For the testing of a COVID-19 procedure that targets the lungs and the breathing system, it is thus appropriate.

In a study passed out, 1,014 affected people tested both for Chest CT and for RT-PCR among 6 January and 6 February 2020. The competence of chest CT in COVID-19 analysis was evaluated with RT-PCR as the situation level. Lively conversion of the RT-PCR test results (negative, positive and negative) was also studied in contrast to serial chest CT scans for affected with multiple RT-PCR examines.

The conclusions revealed a positive RT-PCR result in 601 affected (59\%), while a positive Chest CT scan in 888 (88\%) was existing. Chest CT compassion was $97 \%$ based on the helpful findings of RTPCR as recommended by COVID-19. Medical imaging methods are usually useful ways of analysing COVID-19 and monitoring the movement of diseases. For doctors to judge optimal care approaches, real-time patient nursing is important. Precise and calculable disease studies can help doctors prescribe the accurate medication.

\section{Image based AI Covid-19recognition}

Radiographic arrangement can be accurate, but at once much quicker and less pricey than the PCRtest, via radiological pictures such as chest X-ray (CXR). In accumulation, chest X-rays are lowpriced than other radiological checks, such as CT scans and almost every clinic offers them. CXRbased identification of COVID-19 affected is perceived only to test qualified surgeons, particularly in distant areas, not to be probable at all times. In calculation, the radioscopy of COVID-19 is recent and strange to many professionals who have not had earlier contact with the COVID-19 positive CXRs of affected. So, in order to differentiate COVID-19 positive and negative cases with CXR videos, it is advisable to consider few acquisition algorithms. It's some elementary and cheap deep learning methods. The near-accurate identification of COVID-19 positive affected can be done in a few seconds using this system. The algorithm harmonies to a finest book to mark the synopses precisely to activate the groups. Theoretical acquisitions are made-up to be found in the field of instinctive psychology [5]. Overviewed attainments include the procedure for nervousness of COVID-19.The algorithm acquisition is the subsequent: NB, RF, SVM, CNN, DT and LR.

Since the finest means of identifying COVID-19 is not endorsed for CT scans and X-rays, these may be apposite case-by-case. Imaging can supportestimate the severity of the state in people with risky symptoms. And CT scans or x-rays may be convenient for gauging a patient handling plan whether they are cast-off for lab testing, full health records, and corporeal exams.

\section{Convolutional neural network}

Neural Network (NN) is supposed to be built on the build-up of nodes (called fabricated neurons) that recommendable typical the neurons in the intellect. This scheme repeatedly produces detecting characteristics based on occasions, after some of the above recorded information, after being set. The Algorithm admits as deep learning when it practices various layers of neurons. A Convolutional 
NeuralNetwork (CNN) is a Deep Acquisition algorithm, using an archangel as an input, which authorizes learning weight in the archangel to a range of structures.

In [7] the "transfer acquisitions" in x-ray imaginings of patients with aware bacterial pneumonia, recognized cases of COVID-19, and injected ordinary infections are familiarized in x-ray imaging (with available evidence from a book to improve uneasiness accurate in extra scenario zones. The task aimed to gauge the success of advanced CNN designs for the arrangement of medical archangel.

\section{Logistic regression}

In the circumstance of Logistic Regression, penalized likelihoods have the attraction of creating finite, consistent estimates of regression constraints when the maximum likelihood estimates do not exist due to complete separation. It may be joined with many modules of acclimation saying that an angel acts with various representative chantry [8]. While modest than the CNN, in a hole analysis on the end of the COVID-19 logistic inducement could be activated. The consent given in ROC charge for analytical and CT activities that advise gravitation of COVID-19 was generated for example in [6] logistic enticement. Logistic bribery lessons have shown that the diagnostic causes for severe pneumonia COVID-19 isover 50 years old, chest heaviness, short breath, comorbidity and ahead in the middle around. And COVID-19 all-round X-ray snapshots have been saved from the ResNet152 gadget and once further SMOTE has been improved for the detection of COVID-19 in patients.

\section{Naive Bayes}

The Naive Bayes groupings are among the furthermost basic Bayesian typical designs from the descendants. In grouping with an estimate of the core body, they will offer high levels of attention in the provision of photos in the schema [10]. It was also familiarized for classification in the idea of COVID-19. And outdated statistics and devices have been gathered by the authors in change of CT images for mental functions. The removed presence was again sorted by Naive Bayes mix classification.

\section{Support vector machines}

Vector support machines (SVMs) are overseen methods of reversion, distribution and extra outlier identification for achievement methods. SVM objects to get a hyperplane with an N-dimensional fullness, which simply labels the attributed outcomes. With disputes added, SVM allots an even with the best change between immaterial points of distant groups to the procurement. The vectors backup the hyperplane are such intangible points. These intangible items stimulus the hyperplane place and acclimatization [8].

In [7] it states SVM as one popular ML Algorithm. SVM is greater than other classification models. SVM is used to detect COVID-19 through recognition pictures which are consequential from the chest x-rays. A multi-nearbeginning of the snapshot'sresultant the presence. Also, non-image precises have been used for SVM and synopses for the calculation of COVID-19 cases from backup admission testing. SVM and digests of intake check-ups for emergency states to detect COVID-19 cases. Extracts were attained from 235 affected, 43 percent of whom were COVID-19 cases known.

\section{Unsupervised learning}

As well, the concept of COVID-19 has familiarized unattended achievements. Uninhibited learning, clashed meticulous learning, quest for unknown prototypes in an intellectual stream, and negligible animal intrusion. It types the expectancy densities on the arrivals archetypally available. This system enables it to regulate odd mental genitals that do not suitable with either class and its gear is the body's statistically valued acreage [3]. K-means interest, an entity quantification system, is one of the uninhibited acquirements used via COVID-19. It splits n observations into k groups in which a note for the centenary belongs to the range with the next callous, circumscribed to the array leaders [13]. 


\section{Linear discriminant analysis}

Linear Discriminant Analysis (LDA) is adapted to the procurement of a beeline grouping of occurrences and in order to diagnose and master the gadget, characterizes or separates altar or challenge groups. This resulting collective can be adjusted before the sharing [11] as a beeline classifier for the drop of the region. The function of LDA was to calculate the structures and laws of advances in herpetology in COVID-19 misfortune patients. The after-possessions of the scientific and class trials were analysed and the LDA was altered to alter the haematology.

\section{Random Forest with Decision Trees}

DTs analyse the decisions with respect to the strategy and it leads to final results. RF considerably a result collection of DTs which lead to the final result respective to the outcome. It is about the gathering of the decisions whose significances have been ensured. After a growing insanity because of favouritism, they hold total variance. It is familiarized to assign patient pictures in medical training [12]. And the upper body CT images were familiarized for serious calculation in 176 affected with COVID-19. The inadvertent forest has been demonstrating and doing a virtual assessment of the extent of COVID-19 in affected. The Random Foresttypicallygiveshelpful results. They also validlyadjusted a contagionextent feature as aplan of the broad aggregate of the mixed lungs. In a 3 similar unplanned backwoods arrangement, this extent for contagion was then acclimatised and separated into 4 classes. They adapted five cross endorsement measures to evaluate the performance of the projected algorithm. Added classifiers, for example logistical regression, vector support and neural preparations, were also compared.

\section{Conclusion}

AI is a constanttool for the age-old and exact forecast of COVID-19 and has been dealt with in a prosperity of articles. Most manage their change to the clinical archangel class by ConvolutionalNeural Networks (CNNs). The casual backcountry and support vector apparatuses are managed by few test fallouts. Some are likewise enabled and itfluctuates in the estimation of CT and $\mathrm{x}$-ray metaphors. The inventors of Artificial Intelligence's bravesystems all approve that their system will work fully on calculated truth. However, it is detectable by far that it would now not contempt the skill of an algorithm with appreciation facts for its correspondinglymortified results on the milled. This is since the aptness of adding summaries to the hobby at the outflow of bubbling and totalling artefacts not generally found in homework and judgment reports. Also, the shortening of the annotated routine snap shots is not tolerable. The top two out of 18 study studies in this calculation were permitted for adjusted radiologist extracts. In order to evaluate a huge sum of annotated snaps of COVID-19, the teamwork between clinicians and consultants is fast. Additional abominable in view or abomination, asubstitute to adhere to difficulty may be stimulated to evaluation papers and activated universally as a role of the alterity of skill of a system. The supreme amount of quest researchers used full mock-ups simultaneously with confident well-known copies with some changes. Those with confident modifications that is hardly been made higher confirm the entitlement of increasing mixing models to test complex and more skilledmanners. In calculation, a general adjustment is essential in genetic and/or vaccine finding, collection of calculation and chance valuation for clinical staff. To arrange, COVID-19impulsively has a state of backup in the world's medical system. The reception has shown that this pandemic is truthful in the diversion. Most of the Artificial Intelligenceexecutions were the x-ray neural network (CNN) and CT images.

Since the finest means of identifying COVID-19 is not suggested for CT scans and X-rays, these may be fit case-by-case. Tomography can help gauge the harshness of the disorder in people with lifethreatening symptoms. And CT images or x-rays may be convenient for gauging a patient handling plan whether they are used for lab testing, inclusive health records, and corporeal exams. 


\section{References}

1. WHO Emergencies Coronavirus Emergency Committee Second Meeting. https://www.who.int/docs/defaultsource/coronaviruse/transcripts/ihr-emergency-committee-for-pneumonia-due-to-the-novel-coronavirus-2019ncov-press-briefing-transcript-30012020.pdf?sfvrsn=c9463ac1_2.

2.World Health Organization Novel Coronavirus (2019-nCoV): situation report, 10. https://www.who.int/docs/default-source/coronaviruse/situation-reports/20200130-sitrep-10ncov.pdf?sfvrsn=d0b2e480_2.

3.Ng A (2016) What artificial intelligence can and can’t do right now. In: Harvard Business Review 9. 4.Prince JL, Links JM (2006) Medical imaging signals and systems. Pearson Prentice Hall, Upper Saddle River [ Google Scholar].

5. Mohri M, Rostamizadeh A, Talwalkar A (2018) Foundations of machine learning. MIT Press, Boca Raton [Google Scholar].

6. Narin A, Kaya C, Pamuk Z (2020) Automatic detection of coronavirus disease (covid-19) using x-ray images and deep convolutional neural networks.

7.JS Suri, A Puvvula, COVID-19 pathways for brain and heart injury in comorbidity patients: a role of medical imaging and artificial intelligence-based COVID severity classification: a review.

8. Vapnik Vladimir (2005) Universal learning technology: Support vector machines. NEC J Adv Technol. 2(2):137-144.

9. Li K et al (2020) The clinical and chest CT features associated with severe and critical COVID-19 pneumonia. In: Investigative radiology.

10. Mayo M, Frank E (2020) Improving naive Bayes for regression with optimized artificial surrogate data. Apple Atif Intel. 34(6):484-514.

11. S Manna, J Wruble, SZ Maron, D Toussie, N Voutsinas, M Finkelstein, MA Cedillo, J Diamond, C Eber, A Jacobi, M. Chung. COVID-19: a multimodality review of radiologic techniques, clinical utility, and imaging features. [Google Scholar]

12. Rutkowski L et al (2014) A new method for data stream mining based on the misclassification error. IEEE Trans Neural Netw Learn Syst 26(5):1048-1059

[ Google Scholar].

13. Xia C et al (2020) Distributed K-Means clustering guaranteeing local differential privacy. Comput Secur 90:101699 [ Google Scholar].

S Manna, J Wruble, SZ Maron, D Toussie, N Voutsinas, M Finkelstein, MA Cedillo, J Diamond, C Eber, A Jacobi, M. Chung

COVID-19: a multimodality review of radiologic techniques, clinical utility, and imaging features. 